Cahiers
de a Recherche
surles Droits
Fondamentaux

Cahiers de la recherche sur les droits

fondamentaux

18 | 2020

La vulnérabilité

\title{
La vulnérabilité des migrants
}

Catherine-Amélie Chassin, Alexandra Korsakoff et Laurence MaugerVielpeau

\section{OpenEdition}

\section{Journals}

Édition électronique

URL : https://journals.openedition.org/crdf/6432

DOI : $10.4000 /$ crdf.6432

ISSN : 2264-1246

Éditeur

Presses universitaires de Caen

Édition imprimée

Date de publication : 19 novembre 2020

Pagination : $55-63$

ISBN : 978-2-84133-987-7

ISSN : 1634-8842

Référence électronique

Catherine-Amélie Chassin, Alexandra Korsakoff et Laurence Mauger-Vielpeau, « La vulnérabilité des migrants », Cahiers de la recherche sur les droits fondamentaux [En ligne], 18| 2020, mis en ligne le 19 novembre 2021, consulté le 14 novembre 2022. URL : http://journals.openedition.org/crdf/6432 ; DOI : https://doi.org/10.4000/crdf.6432 


\title{
La vulnérabilité des migrants
}

\author{
Catherine-Amélie CHASSIN \\ Maître de conférences (HDR) en droit public à l'université de Caen Normandie \\ Centre de recherche sur les droits fondamentaux et les évolutions du droit (CRDFED, EA 2132)
}

Alexandra KORSAKOFF

Docteure en droit public de l'université de Caen Normandie

Centre de recherche sur les droits fondamentaux et les évolutions du droit (CRDFED, EA 2132)

\section{Laurence MAUGER-VIELPEAU}

Professeure de droit privé à l'université de Caen Normandie

Institut Demolombe (EA 967)

I. La vulnérabilité induite par la migration

A. La vulnérabilité de l'État face à la migration

1. La migration, conséquence d'un conflit

2. La migration, cause de conflits

B. La migration et la vulnérabilité du migrant

1. La rupture sociale du migrant: les diasporas

2. La rupture juridique du migrant

II. La reconnaissance du statut de réfugié aux femmes persécutées

A. La problématique de l'auteur des persécutions

1. La remise en cause des persécutions étatiques

2. Le défi de l'asile interne

B. La problématique des motifs de persécution

1. La faible mobilisation des motifs " traditionnels"

2. Le recours au motif de l'appartenance à un certain groupe social

Dans le vocabulaire courant, le terme «migrant» signifie «qui participe à une migration, spécialement travailleur originaire d'une région peu développée, s'expatriant pour trouver du travail, ou un travail mieux rémunéré (émigrant, immigrant)». Le nom «migration» renvoie notamment au «déplacement de populations qui passent d'un pays dans un autre pour s'y établir (émigration, immigration, exode, invasion) " et au "déplacement de populations d'un endroit à un autre (migrant) $»^{1}$. La première partie abordera la vulnérabilité des migrants du point de vue de l'État et s'interrogera sur la vulnérabilité du migrant, quels que soient son âge et son identité

1. Le nouveau petit Robert: dictionnaire alphabétique et analogique de la langue française, Paris, Le Robert, 1996. 
sexuelle, confronté à toutes sortes de ruptures mais aussi sur la migration comme facteur de vulnérabilité de l'État lui-même. La seconde partie mettra en lumière des victimes particulières que sont les femmes persécutées dans leur pays d'origine et qui cherchent protection auprès de la France en réclamant le statut de réfugié, lequel est bien difficile à obtenir tant la définition du réfugié demeure rédigée en des termes restrictifs en droit français.

\section{La vulnérabilité induite par la migration ${ }^{2}$}

En 1948, le sociologue américain Eugene Kulischer pointait:

L'histoire de l'humanité est une histoire d'errements. Certaines époques des plus reculées ont souvent été désignées par le terme ère des grandes migrations. Cette terminologie laisse présumer qu'à d'autres périodes les mouvements migratoires ont été plus séminaires, notamment avec la sédentarisation des populations. Mais en réalité, aucune population n'a jamais été immobile. Chaque époque est une période de grande migration ${ }^{3}$.

L'humanité est une masse migrante, par essence. L'érection progressive des frontières est venue limiter la praticabilité du phénomène; elle n'a pour autant pas mis un terme aux migrations. Tout au plus a-t-elle rendu plus visible le passage d'une souveraineté à une autre. Là où l'accueil d'un étranger pouvait être un facteur souhaité par la seigneurie, le nouveau venu venant accroître la richesse financière et les troupes mobilisables ${ }^{4}$, l'affirmation des souverainetés étatiques est venue construire des barrières parfois insurmontables. Et pourtant les migrations se poursuivent, qu'elles soient contraintes par un événement extérieur (persécution ou guerre) ou qu'elles répondent à un besoin économique, selon l'adage qui résume la migration du travail: ubi lucrum, ibi patria («Là où est l'argent se trouve ma patrie») $)^{5}$. Au sein de l'Union européenne, ces migrations sont même devenues d'une grande banalité du fait du libre établissement, qui permet aux étudiants de bénéficier sans entraves du programme Erasmus+ ou aux agents des États membres de rejoindre les bureaux de Bruxelles ou Luxembourg - sans oublier les retraités qui préfèrent les rivages du Sud de l'Europe aux brouillards incertains du Nord.
Évoquer la migration impose de dépasser les clichés: le migrant n'est pas nécessairement le ressortissant d'un État d'Afrique qui tente sa chance sur un navire à la flottabilité douteuse entre la Libye et Malte; il peut être autre qu'un Syrien essayant d'échapper à une guerre interminable. Du point de vue du droit international, le migrant se définit comme toute personne qui franchit une frontière en vue de s'installer pour plus de trois mois sur le territoire d'un État dont il n'a pas la nationalité. Sont donc visés certes les cas sus-évoqués, mais aussi les étudiants, les sportifs de haut niveau, les milliardaires décidant d'investir dans un paradis (fiscal ou non).

Les profils des migrants sont variés. Mais tous partagent un point commun: ils ont quitté leur État. Or la migration n'est jamais anodine. Ceux qui ont vécu une expérience d'expatriation, fut-ce de quelques mois, le savent: il n'est pas facile de partir; il n'est pas non plus facile de revenir. Le migrant se trouve de facto dans une forme de vulnérabilité (B). Mais cette vulnérabilité existe aussi pour l'État, certes celui d'origine, mais aussi l'État d'accueil (A). Ce sont ces deux aspects qui guideront nos réflexions.

\section{A. La vulnérabilité de l'État face à la migration}

La migration n'est jamais anodine, ce point ne sera jamais suffisamment souligné. Les États ont donc tenté d'organiser ces migrations dès 1919, lors de l'adoption du traité de Versailles dont la partie XIII crée l'Organisation internationale du travail (OIT). Le préambule de la constitution de l'OIT souligne le lien indéfectible entre les conditions du travail et la paix universelle, la frustration induite par les premières mettant en danger la seconde ${ }^{6}$. L'effort d'organisation des migrations du travailleur se développe dans le cadre de l'OIT', mais aussi dans le cadre des Nations unies avec l'adoption d'une convention propre ${ }^{8}$ et, surtout, du Pacte mondial pour des migrations sûres, ordonnées et régulières ${ }^{9}$.

Pourtant le défi de la migration demeure. Car au-delà de ses modalités, la migration n'est pas sans conséquences sur la stabilité de certains États, voire de certaines régions du monde: elle peut être la conséquence (1) ou la cause de conflits (2).

2. Par Catherine-Amélie Chassin.

3. E. Kulischer, Europe on the Move. War and Population Changes, 1917-1947, New York, Columbia University Press, 1948, p. 8 (nous soulignons). Sauf mention contraire, toutes les traductions sont des auteures.

4. Voir J.-A. Roux, «L'entraide des États dans la lutte contre la criminalité», Recueil des cours de l'Académie de droit international, vol. 36,1931, p. 82.

5. Selon le mot de J. T. Shuval, "Diaspora Migration: Definitional Ambiguities and a Theorical Paradigm», International Migration Quaterly Review, vol. $38, \mathrm{n}^{\circ}$ 5, 2000, p. 47.

6. Constitution de l'OIT, préambule: «[...] il existe des conditions de travail impliquant pour un grand nombre de personnes l'injustice, la misère et les privations, ce qui engendre un tel mécontentement que la paix et l'harmonie universelles sont mises en danger, et [...] il est urgent d'améliorer ces conditions $[\ldots] »$.

7. Voir la convention OIT nº 97, Genève, $1^{\text {er }}$ juillet 1949, complétée par la convention OIT no 143 , Genève, 4 juin 1975.

8. Organisation des Nations unies, Convention internationale sur la protection des droits de tous les travailleurs migrants et des membres de leur famille, New York, 18 décembre 1990, Recueil des traités des Nations unies, vol. 2220, 2004, p. 3.

9. Le Pacte mondial pour des migrations sûres, ordonnées et régulières fait suite à l'adoption de l'Agenda 2030 (Assemblée générale des Nations unies, résolution 70/1 du 25 septembre 2015). Le pacte lui-même, dénué de valeur obligatoire, est adopté par les États à Marrakech le 10 décembre 2018. 


\section{La migration, conséquence d'un conflit}

Hannah Arendt met en exergue ce lien entre migration de masses humaines et paix:

Les guerres civiles qui ont inauguré et marqué les années vingt d'une paix incertaine n'ont pas seulement été plus cruelles et plus sanglantes que les précédentes; elles ont entraîné l'émigration de groupes $[\ldots]^{10}$.

Sont ici visés ceux qui pourraient être qualifiés de migrants du désespoir, contraints de quitter leurs foyers pour échapper à des persécutions que leur État de nationalité ne peut ou ne veut empêcher. L'afflux des personnes contraintes de quitter leur pays a conduit la communauté internationale, au sortir de la Première Guerre mondiale, à laisser émerger une nouvelle branche du droit international: le droit des réfugiés. Il ne s'agit pas ici de reprendre la succession des textes depuis les premiers Arrangements relatifs aux réfugiés russes (1922) et arméniens (1924); le texte phare reste aujourd'hui la Convention de Genève sur le statut international des réfugiés (1951) complétée par plusieurs textes à vocation régionale en Afrique (1969), en Amérique latine (1984) et dans l'Union européenne (2004). Ces textes sont essentiels en ce qu'ils créent un statut reconnu au plan international (statut de réfugié) ou régional - laissant aux États le soin d'en déduire, dans leurs ordres internes, le droit sans doute le plus essentiel pour celui qui a dû fuir: l'asile, c'est-à-dire un droit au séjour. Car le bénéfice d'une protection internationale ou régionale n'entraîne pas de plein droit un séjour pour l'intéressé, les États ayant toujours refusé de se lier sur une prérogative indéfectible de leur souveraineté: la gestion du séjour des étrangers sur leur territoire. Il est donc envisageable de voir un réfugié privé du droit au séjour régulier sur le territoire de l'État qui lui a reconnu le statut ${ }^{11}$ - sans pour autant que l'on ne puisse l'éloigner vers l'État dont il provient, par application du principe dit de non-refoulement (Convention de Genève, art. 33, $\$ 1^{\text {er } 12}$ ).

Le droit international des réfugiés est conçu comme un droit de protection des individus. Essentiel, cet instrument juridique qu'est la Convention de Genève de 1951 est pourtant dépassé face aux arrivées massives. Ainsi lors du génocide rwandais, en 1994, le Haut Commissariat des Nations unies pour les réfugiés (HCR) dénombre plus de deux millions de personnes qui fuient dans les États limitrophes ${ }^{13}$. Dans le cadre de la crise syrienne, les statistiques délivrés par le HCR ${ }^{14}$ évoquent la présence de près d'un million de réfugiés au Liban, pour un pays qui compte au total six millions d'habitants - et qui, faut-il le rappeler, a déjà dû absorber l'arrivée des réfugiés venus de Palestine en 1948 et 1967. En Turquie, ils étaient, en février 2020, environ 3,6 millions de réfugiés syriens. Cette présence massive est devenue une arme politique aux mains du gouvernement, le président Erdogan n'hésitant pas, dès octobre 2019 alors que ses troupes entraient en Syrie, à menacer d'ouvrir les portes si l'Union européenne ne soutenait pas ses initiatives militaires ${ }^{15}$.

Cette instrumentalisation des réfugiés rappelle ainsi que les migrations peuvent, aussi, être cause de conflits.

\section{La migration, cause de conflits}

L'actuel secrétaire général des Nations unies souligne l'impact des migrations sur la paix mondiale:

Croissance de la population. Urbanisation. Raréfaction de l'eau. Insécurité alimentaire et énergétique. Volatilité des prix des matières premières.

Lorsque nous regardons l'avenir, il semble certain que ces tendances vont continuer à s'accroître et à se tendre, créant ainsi une concurrence accrue et des conflits relatifs à des ressources naturelles de plus en plus rares. En conséquence, on peut s'attendre à voir croître le nombre de personnes contraintes de quitter leur communauté, leur pays ou leur continent ${ }^{16}$.

Le phénomène migratoire risque en effet d'exacerber des tensions, déstabilisant des États. Ce lien a été établi en 1989 par Peter H. Gleick ${ }^{17}$, dont les travaux montrent que des arrivées massives viennent pressurer les ressources existantes, et peuvent agir comme un détonateur pour des conflits sous-jacents ${ }^{18}$. Commence alors un cercle infernal, tant pour les État que pour les populations: la rareté des ressources crée de nouvelles migrations, lesquelles vont à leur tour créer de nouveaux conflits. Ce scénario est notamment dépeint par deux géographes australiens, Jon Barnett et Neil Adger ${ }^{19}$. Se concentrant sur la raréfaction

10. H. Arendt, Les origines du totalitarisme. 2. L'impérialisme [The Origins of Totalitarianism, 1951], M. Leyris (trad.), Paris, Fayard (Points. Essais), 1997 , p. 240.

11. Voir, par exemple, CJUE, 24 juin 2015, H. T., C-373/13; CJUE, 14 mai 2019, M. et autres, C-391/16.

12. Organisation des Nations unies, Convention relative au statut des réfugiés, Genève, 28 juillet 1951, Recueil des traités des Nations unies, vol. 189, 1954, p. 177.

13. Haut Commissariat pour les réfugiés des Nations unies, Les réfugiés dans le monde: cinquante ans d'action humanitaire, Genève - Paris, HCR - Autrement, 2000, p. 247.

14. Voir les chiffres actualisés à partir de la page Internet: www.unhcr.org/fr/urgence-en-syrie.html.

15. Le 10 octobre 2019, le président turc est très clair dans l'instrumentalisation de ces réfugiés syriens à l'heure où il déploie l'opération Source-de-Paix: "Europe, si tu qualifies notre opération militaire de "mouvement d'occupation", notre travail sera facile: nous ouvrons les portes et t'envoyons 3,6 millions de réfugiés » (cité par A. Mourenza, correspondant à Istanbul du journal espagnol El pais, édition en ligne du 11 octobre 2019: https:// elpais.com/internacional/2019/10/10/actualidad/1570709328 486074.html)

16. A. Guterres, "Nansen Conference on Climate Change and Displacement», Oslo, 6 juin 2011, en ligne: http://www.unhcr.org/4deffffb9.html.

17. P. H. Gleick, "The Implication of Global Climatic Changes for International Security», Climatic Change, vol. 15, 1989, p. 309-325.

18. Voir aussi R. McLeman, "Climate Change, Migration and Critical International Security Considerations", IOM Migration Research Series, $\mathrm{n}^{\circ}$ 42, 2011, p. 25 sq.; M. Beniston, "Climate Change and Its Impact: Growing Stress Factors for Human Societies», Revue internationale de la Croix-Rouge, vol. 92, n 879, 2010, p. 557-568.

19. Voir J. Barnett, W. N. Adger, «Climate Change, Human Security and Violent Conflict», Political Geography, vol. 26, nº 6, 2007, p. 639-655. 
des ressources, ils analysent les conflits qui ont ravagé le monde entre 1930 et 2007 , et recensent trente-huit migrations induites par des bouleversements climatiques. Dans la moitié des cas, cette migration a causé un conflit armé dans le pays d'accueil. Ils citent en particulier les pays de la Corne de l'Afrique: la désertification rampante a conduit les populations à migrer, à la recherche d'eau et de nourriture. Ce mouvement a intensifié les tensions dans les pays voisins, sur un terreau fragilisé par des rivalités ethniques. Ces travaux démontrent que la vulnérabilité des États face à la migration des masses n'est pas un mythe.

Cette analyse ne saurait se limiter aux États émergents: la vulnérabilité des États face au défi de l'assistance humanitaire ne saurait être sous-estimée. On peut songer ici à la charge de l'accueil des migrants pour des États européens comme la Grèce ou Malte, mais aussi aux enjeux pour des États tels la Colombie et le 1,3 million de Vénézuéliens qui se trouvent sur son territoire en 2019-202O, ou encore la Jordanie et le Liban face aux réfugiés syriens. À cette pression économique et structurelle peut être joint le risque réel d'un basculement politique; souvenons-nous qu'en 2016, l'élection de Donald Trump à la présidence américaine est due, pour partie au moins, au fantasme entretenu d'une invasion des États-Unis par des Mexicains et à la nécessité, pour s'en prémunir, de construire un mur infrangible entre ces deux États.

Indéniablement, les migrations sont un enjeu pour les États, car elles soulignent leurs vulnérabilités. Elles sont un enjeu non moins fondamental pour les individus.

\section{B. La migration et la vulnérabilité du migrant}

Habituellement les individus sont attachés à un État qu'ils ne quitteront que ponctuellement au cours de leur vie, un attachement juridique à travers le lien de nationalité, mais aussi un attachement affectif qui permet de considérer que «l'individu est ancré dans son État comme un cèdre du Liban $»^{20}$. La migration lui fait quitter cet État d'origine pour s'installer dans un autre territoire, ce qui crée pour l'individu une sorte de dualité, entre son État d'origine et l'État d'accueil. Cette situation aboutit à une double rupture, sociale (1) et juridique (2).

\section{La rupture sociale du migrant: les diasporas}

Le migrant est entre deux mondes: son État d'origine, dont il a les codes socioculturels et dont il connaît la plupart des ressorts politiques, et l'État d'accueil, dans lequel il souhaite le plus souvent prendre une place. Or l'un des effets de la migration est qu'elle fait perdre les repères dans l'État d'origine, sans pour autant permettre une intégration automatique dans la société de l'État d'accueil.

La rupture vis-à-vis de l'État d'origine peut rapidement aboutir à un «déracinement » du migrant ${ }^{21}$. Ce migrant va être amené à reconstruire une image de son pays d'origine, image souvent embellie et territorialisée en ce sens que chaque diaspora va, compte tenu de sa propre évolution au sein du pays d'accueil dans lequel elle se trouve, recréer un pays d'origine qui ne correspond que partiellement à la réalité $^{22}$. Chaque diaspora, chaque migrant, va ainsi imperceptiblement perdre pied avec la réalité de l'État d'origine, auquel pourtant il entendra souvent se rattacher au moins en partie. Nous pourrions prendre un exemple simple mais parlant pour ceux qui, ces dernières années, vivent en France: les acronymes de ZAD («zone à défendre») ou de LBD («lanceur de balles de défense») sont devenus communs dans la presse; pourtant celui qui a quitté la France pour s'installer ailleurs, même s'il revendique son attachement à son pays d'origine, n'aura pas nécessairement suivi l'émergence de ces notions. L'exemple est certes anecdotique, mais il montre le mouvement de rupture entre le migrant et son État d'origine. Ce mouvement avait déjà été théorisé par Hannah Arendt, s'agissant de la souffrance des déracinés ${ }^{23}$, que l'on appellerait aujourd'hui des migrants contraints: elle souligne la perte du domicile et des habitudes quotidiennes, la perte de la langue maternelle comme expression spontanée des sentiments, l'illusion d'oublier le passé.

Cette rupture, inéluctable et qui s'accentue avec le temps, peut même devenir un véritable divorce dans la mesure où le cheminement du migrant est parallèle à celui de son État d'origine, qui va se développer en dehors de son ressortissant. Le migrant peut alors en arriver à mépriser son pays d'origine, estimant qu'il a trahi la société dont le migrant a cru garder la mémoire ${ }^{24}$. Le retour éventuel du migrant peut alors être difficile, d'autant plus difficile que les populations demeurées dans le pays d'origine pourront avoir une forme de jalousie vis-à-vis de cet expatrié parfois enrichi, et qui revient au pays en jugeant de son évolution en son absence ${ }^{25}$.

Cette rupture ne serait pas nécessairement problématique, si elle s'accompagnait de l'admission dans une autre communauté nationale. Or tel n'est pas le cas: le migrant reste un étranger aux yeux de l'État d'accueil. Les

20. Y. Benziman, «Citizenship as a Home», in Plurality and Citizenship in Israel, D. Avnon, Y. Benziman (dir.), Londres, Routledge, 2010, p. 53-67.

21. A. A. Cançado Trindade, "Le déracinement et la protection des migrants dans le droit international des droits de l'homme», Revue trimestrielle des droits de l'homme, vol. 19, $\mathrm{n}^{\circ}$ 74, 2008, p. 289-328. L'expression est reprise de son opinion dissidente émise dans l'affaire de la Cour interaméricaine des droits de l'homme, 15 juin 2005, Communauté Moiwana c. Surinam, série C, nº 124.

22. W. Safran, "Comparing Visions of the Nation. The Role of Ethnicity, Religion and Diaspora Nationalism in Armenian, Jewish and Sikh Relations to the Homeland», in Nationalism in a Global Era. The Persistence of Nations, M. Young, E. Zuelow, A. Sturm (dir.), Londres - New York, Routledge, 2007, p. 33-54.

23. H. Arendt, La tradition cachée [Die verborgene Tradition, 1976], S. Courtine-Denamy (trad.), Paris, C. Bourgois, 1987, p. 58 et p. 125

24. J. T. Shuval, «Diaspora Migration...», p. 46

25. Voir, par exemple, R. Schwartz, "Sponsors or Spoilers: Diasporas and Peace Processes in the Homeland", in The Failure of the Middle East Peace Process? A Comparative Analysis of Peace Implementation in Israel/Palestine, Northern Ireland and South Africa, G. Ben-Porat (dir.), Basingstoke - New York, Palgrave Macmillan, 2008, p. 135-154. 
conséquences de la qualification juridique sont lourdes. Elle renvoie à toute la réglementation relative à l'entrée sur le territoire (en France, art. L. 211-1 sq. du Code de l'entrée et du séjour et du droit d'asile - CESEDA), marquant à chaque passage de la frontière que l'étranger n'est pas un résident comme les autres, et qu'il ne peut entrer qu'en raison d'une décision de l'autorité publique territorialement compétente. De la même façon, l'étranger a l'obligation de détenir un titre de séjour qui matérialise le droit concédé par l'État de demeurer sur son territoire. Là encore, en France la réglementation est fixée par le CESEDA, qui prévoit tout à la fois la compétence de l'autorité préfectorale en fonction des motifs du séjour (art. L. 311-1 sq.), la possibilité de contrôle de ce titre de séjour (art. L. 611-1 sq.) et la sanction de ce titre à travers la possibilité d'éloignement du territoire (art. L. 511-1 sq.). Faut-il le rappeler: le droit international garantit effectivement le droit de séjour dans son propre pays, principe consacré par la plupart des conventions relatives aux droits de l'homme et qui relève du droit coutumier ${ }^{26}$. Mais cette obligation ne concerne que les seuls nationaux, et non les étrangers.

Chaque renouvellement, chaque contrôle permet ainsi de souligner la fragilité de la condition d'étranger. Apparaît ainsi subrepticement, aux côtés de la rupture sociale, une rupture juridique.

\section{La rupture juridique du migrant}

L'existence d'un code spécifiquement dédié aux étrangers (le CESEDA, en France) trahit l'existence de fêlures juridiques, dans la mesure où le migrant, quoique titulaire des droits fondamentaux de la personne humaine parce que personne humaine, se trouve pourtant privé de certains de ces droits.

La rupture juridique est, d'abord, une rupture politique; à raison, le migrant a pu être qualifié d' « orphelin de l'État ${ }^{27}$. L'expatriation devient source de vulnérabilité sur le plan politique: ainsi peut-on songer ici à ces ressortissants britanniques installés depuis plus de quinze ans sur le territoire d'un autre État membre et qui, du fait du Brexit, non seulement ne votent plus sur le territoire de cet État membre mais ne votent plus non plus au Royaume-Uni.

La rupture juridique existe également s'agissant de la vie familiale. Si la famille est "l'élément naturel et fondamental de la société ${ }^{28}$, si des juges ont pu reconnaître le caractère sacré de la famille, la notion même de famille est volatile car imprégnée largement des conceptions culturelles de l'État d'origine. C'est alors «au droit international privé qu'il faut demander comment concilier respect des droits de l'homme tels que les conçoit l'ordre juridique du for et respect des cultures étrangères ${ }^{29}$. En France, ce prisme est prégnant dans les questions touchant au regroupement familial, tel qu'organisé par le CESEDA (art. L. 411-1sq.). Le migrant va devoir adapter sa vie familiale aux critères français. La famille est ici limitée aux couples mariés sous réserve entre autres que le mariage ne concerne pas des sous-âgés (art. L. 411-1) et ne soit pas polygamique (art. L. 411-7); seuls les enfants mineurs au jour du dépôt de la demande peuvent être accueillis dans ce cadre (art. R. 411-3), quelle que soit la nature du lien de filiation ${ }^{30}$. Il a fallu que le juge vienne compléter la loi pour admettre d'intégrer dans cette famille les enfants mineurs accueillis par voie de kafala : retenue par le CESEDA s'agissant des enfants du conjoint (art. L. 411-3) et par l'accord franco-algérien de 1968 amendé en 2001 pour les enfants de nationalité algérienne (Titre II modifié), c'est le juge administratif qui a permis l'évolution en ce domaine. Par l'affaire Hocini de $2010^{31}$, le Conseil d'État considère en effet que, s'agissant d'enfants accueillis par décision judiciaire prononcée dans un État de droit musulman, le critère permettant de rattacher l'enfant au demandeur peut être certes le lien de filiation, mais aussi la délégation parentale constituée par cette décision de kafala. Le juge s'appuie alors sur l'intérêt supérieur de l'enfant, ce qui permet d'étendre cette solution à l'ensemble des mineurs et non aux seules hypothèses visées par la loi française et le traité franco-algérien. Pour autant, la Cour de justice de l'Union européenne elle-même le souligne: la kafala ne saurait être assimilée à une adoption ${ }^{32}$. Il en résulte que la famille telle qu'elle existait dans le pays d'origine ne sera pas nécessairement la famille autorisée dans le pays hôte.

Ce simple fait pointe toute la vulnérabilité du migrant pris dans son individualité. Mais les exemples pourraient être multipliés, au-delà de la famille: la vulnérabilité sera renforcée par la question linguistique, mais aussi par la nécessité de faire reconnaître les éventuels diplômes et qualifications professionnelles. Il y a bien une fragilité propre au migrant, qu'il soit travailleur ou étudiant, enfant ou malade, homosexuel ou militant d'une cause politique propre, qu'il soit d'une couleur ou d'une religion minoritaires dans l'État d'accueil, qu'il soit femme ou handicapé. Ce sont en réalité toutes les vulnérabilités de l'être humain qui sont ici renforcées par le déracinement de l'intéressé.

26. Hans Kelsen lui-même avait rappelé que l'État de nationalité a toujours l'obligation d'accepter son ressortissant (H. Kelsen, «Théorie générale du droit international public: problèmes choisis ", Recueil des cours de l'Académie de droit international, vol. 42, 1932, p. 254)

27. M. Denis-Linton, conclusions sur l'arrêt CE, Ass., 2 décembre 1994, $D^{\text {me }}$ Agyepong, Revue française de droit administratif, 1995, p. 86-94, spéc. p. 9o.

28. L'expression est utilisée par le Pacte international relatif aux droits civils et politiques du 1o décembre 1966, art. 23; voir aussi Cour interaméricaine des droits de l'homme, avis consultatif, 28 août 2002, Statut juridique et droits des enfants, série A, n ${ }^{\circ} 17, \$ 66$.

29. H. Gaudemet-Tallon, "Le pluralisme en droit international privé, richesses et faiblesses (le funambule et l'arc-en-ciel)», Recueil des cours de l'Académie de droit international, vol. 312, 2005, p. 396.

30. Ce qui inclut dès lors les enfants accueillis par voie d'adoption: voir CE, 6 décembre 2002, Lukundu, tables du Recueil Lebon, p. 588 .

31. CE, $1^{\text {er }}$ décembre 2010, $M^{\text {me }}$ Naili ép. Hocini, $\mathrm{n}^{\mathrm{o}} 328063$.

32. CJUE, 26 mars 2019, S. M., C-129/18. 


\section{La reconnaissance du statut de réfugié aux femmes persécutées ${ }^{33}$}

Certes la migration peut être source de vulnérabilités, mais elle peut également en constituer un remède. Sont ici visés les étrangers qui fuient une situation de vulnérabilité acquise dans leur pays d'origine et se réclament du statut de réfugié en France. Mais les textes qui régissent la reconnaissance d'une telle protection restent entachés d'insuffisances, de telle sorte qu'ils ne permettent pas de se saisir de l'ensemble des situations de vulnérabilité. Et tel n'est notamment pas le cas des femmes qui sont persécutées parce que ce sont des femmes dans leur pays d'origine.

Rappelons que la notion de réfugié a été définie sur la scène internationale après la Seconde Guerre mondiale. Aux termes de l'article $1, \$$ A.2 de la Convention de Genève du 28 juillet 1951, il s'agit d'un individu qui craint d'être persécuté dans son pays d'origine du fait de sa race, religion, nationalité, opinions politiques ou appartenance à un certain groupe social ${ }^{34}$. Cette définition a été complétée, en France, par un sixième motif de persécution. En ce sens, la loi no 98-349 du 11 mai 1998 prend acte de l'alinéa 4 du préambule de la Constitution du 27 octobre 1946 en ouvrant également le statut de réfugié aux personnes persécutées dans leur pays d'origine en raison de leur action en faveur de la liberté.

Cette définition du réfugié ne mentionne pas l'identité sexuelle, si bien que les théoriciennes féministes du droit l'ont remise en cause à partir des années 1980, en se fondant sur l'évolution du contexte juridique ${ }^{35}$. En effet, si les années 1950 étaient caractérisées par une certaine indifférence à l'égard des droits des femmes, ces derniers ont été revalorisés au cours des années 1970. L'intervention de la Convention sur l'élimination de toutes les formes de discrimination à l'égard des femmes en date du 18 décembre 1979 en est un exemple topique. Or, «tout instrument international doit être interprété et appliqué dans le cadre de l'ensemble du système juridique en vigueur au moment où l'interprétation a lieu $»^{36}$. Il convient donc de prendre en compte les évolutions consenties au sein du corpus du droit international et européen des droits de l'homme pour redéfinir ce qu'est un réfugié, et y inclure les femmes persécutées.

Ces critiques ont trouvé un écho sur la scène européenne dès 1984, avec une résolution du Parlement européen, par laquelle il prie les États de reconnaître la qualité de réfugié «aux femmes qui, dans certains pays, sont victimes de traitements cruels ou inhumains parce que l'on considère qu'elles ont enfreint des règles morales ou éthiques de la société où elles vivent ${ }^{37}$. Le Haut Commissariat aux réfugiés ${ }^{38}$ et le Conseil de l'Europe ${ }^{39}$ suivent dès 1985. Et depuis cette date, on a vu fleurir nombre de recommandations, voire des dispositions contraignantes du droit de l'Union européenne ${ }^{40}$, dans le même sens. Mais le législateur français reste sourd à ces développements, de telle sorte que ce sont les juridictions administratives qui se sont chargées de faire évoluer la définition du réfugié en faveur des femmes persécutées, grâce à leur seul pouvoir d'interprétation des textes.

Il convient donc de revenir ici sur ces évolutions, mais également leurs insuffisances, car force est de constater qu'elles ne permettent toujours pas à l'ensemble des femmes persécutées dans leur pays d'origine de se voir reconnaître la qualité de réfugié en France.

Les deux principales critiques que les théoriciennes féministes du droit ont adressées à la définition du réfugié seront successivement analysées, concernant d'abord l'auteur (A) puis les motifs (B) de persécution.

\section{A. La problématique de l'auteur des persécutions}

À l'origine, seules les atteintes aux droits perpétrées par un État sont assimilables à des persécutions, et par suite susceptibles d'ouvrir droit au statut de réfugié. Cette lecture, décriée, a depuis été abandonnée (1), mais la prise en compte des atteintes aux droits réalisées par des particuliers reste encore imparfaite (2).

\section{La remise en cause des persécutions étatiques}

Initialement, la notion de persécution recouvre les seules atteintes graves aux droits commises par un agent de

33. Par Alexandra Korsakoff.

34. Organisation des Nations unies, Convention relative au statut des réfugiés, p. 153.

35. D. Indra, "Gender: A Key Dimension of the Refugee Experience», Refuge, vol. 6, n 3, 1987, p. 3-4; N. Kelly, "Gender-Related Persecution: Assessing the Asylum Claims of Women ", Cornell International Law Journal, vol. 26, n 3, 1993, p. 625-674; A. Macklin, «Refugee Women and the Imperative of Categories», Human Rights Quarterly, vol. 17, nº 2, 1995, p. 213-277.

36. Cour internationale de justice, «Conséquences juridiques pour les États de la présence continue de l'Afrique du Sud en Namibie (Sud-Ouest africain) nonobstant la résolution 276 (1970) du Conseil de Sécurité», avis consultatif du 21 juin 1971, Recueil, 1971, p. 31, $\$ 53$. Ce principe fait écho à l’article 31, \$ 3.c de la Convention de Vienne sur le droit des traités, conclue à Vienne le 23 mai 1969, Recueil des traités des Nations unies, vol. 1155, 1987, p. 331.

37. Parlement européen, résolution sur l'application de la Convention de Genève relative au statut des réfugiés, 13 avril 1984, Journal officiel des Communautés européennes, C 127, 14 mai 1984, p. 137, \$1-2.

38. Comité exécutif du Haut Commissariat aux réfugiés, conclusions $\mathrm{n}^{\circ} 39$ (XXXVI) sur les femmes réfugiées et la protection internationale, 18 octobre 1985 , point $\mathrm{k}$.

39. Assemblée parlementaire du Conseil de l'Europe, recommandation 1261 (1995) sur la situation des femmes immigrées en Europe, 15 mars 1995 , $\$ 9.12$

40. Sur ce point, lire les directives 2004/83/CE et 2011/95/UE, respectivement en date des 29 avril 2004 et 13 décembre 2011, dites "Qualifications" (Journal officiel de l'Union européenne, L 304, 30 septembre 2004, p. 12-23 et Journal officiel de l'Union européenne, L 337, 20 décembre 2011, p. 9-26). 
l'État ${ }^{41}$ qui n'agit pas à titre personnel ${ }^{42}$. Cette interprétation a été très critiquée au motif que de nombreuses violences subies par les femmes émanent de particuliers, et non des autorités étatiques, ce qui a pour conséquence de les placer en dehors du champ de la définition du réfugié ${ }^{43}$. C'est la raison pour laquelle elle a progressivement été remise en cause, en trois temps.

Tout d'abord, en 1983, dans sa jurisprudence Dankha, le Conseil d'État accepte d'assimiler les actes commis par des particuliers à des persécutions lorsqu'ils ont été perpétrés avec l'encouragement ou la tolérance volontaire des autorités ${ }^{44}$. Cette évolution a, par exemple, permis l'élection au statut des ressortissantes algériennes défendant les droits des femmes et persécutées par des intégristes religieux au cours des années $1990^{45}$.

Puis, en 1991, dans la jurisprudence Arthur, la juridiction administrative accepte de qualifier de persécutions, les actes commis par des autorités de fait qui ont supplanté les autorités étatiques sur une portion du territoire ${ }^{46}$. Cette jurisprudence a en particulier été appliquée à la situation de l'Afghanistan après la chute du président Rabbani en 1994. Elle a permis la reconnaissance du statut à des demandeuses persécutées par des moudjahidines en raison de leurs positions sur la condition des femmes ${ }^{47}$.

Il faut finalement attendre la loi $\mathrm{n}^{\circ} 2003-1176 \mathrm{du}$ 10 décembre 2003 pour que l'exigence de l'auteur étatique de persécution soit complètement abandonnée. Depuis, peu importe l'auteur des persécutions. L'article L. 713-2 du CESEDA prévoit expressément que les atteintes graves aux droits peuvent constituer des persécutions qu'elles soient commises par des agents étatiques ou non étatiques.

On pourrait donc à première vue croire que la critique des théoriciennes féministes du droit est complètement dépassée. Mais tel n'est pas le cas. En effet, parallèlement à l'abandon du critère de l'agent étatique de persécution, on a vu apparaître une restriction, afin de limiter la prise en compte de ces «nouvelles» persécutions justement. Il s'agit de la possibilité de réinstallation interne, plus communément appelée «asile interne».

\section{Le défi de l'asile interne}

L' « asile interne» consiste à tenir compte de la possibilité de réinstallation d'un demandeur sur une autre partie du territoire de son pays d'origine pour tenir en échec sa demande de statut de réfugié. Cette notion, issue des décisions Dzebric et Dujic de la Commission des recours des réfugiés (CRR) en date du 12 février $1993^{48}$, a été entérinée par l'article $1^{\text {er }}$ de la loi du 10 décembre 2003 précitée, lequel précise qu'il convient de tenir compte de la nature de l'agent de persécution pour l'appliquer. En particulier, les travaux parlementaires indiquent qu'elle ne peut être mobilisée que dans l'hypothèse d'une persécution commise par des particuliers. En d'autres termes, si la loi de 2003 a certes permis de prendre en compte les persécutions commises par des particuliers, elle a en même temps consacré un obstacle à leur prise en compte: la possibilité de réinstallation interne du demandeur.

Il faut tout de même souligner que la notion d'asile interne n'est pas systématiquement mobilisée dans ce contentieux: d'une part, parce que la loi affirme que c'est une simple faculté, et non une obligation ${ }^{49}$, et d'autre part, parce que son application a été strictement encadrée. En effet, la possibilité de réinstallation interne d'un demandeur ne peut faire échec à une demande de statut de réfugié que sous certaines conditions, relatives à l'installation et au séjour dans la zone de refuge. Tout d'abord, le requérant doit pouvoir s'y installer légalement et en toute sécuritéso. Puis, il doit pouvoir y séjourner durablement ${ }^{51}$ et " $y$ mener une existence normale ${ }^{52}$, c'est-à-dire que la réinstallation ne doit pas entraîner un "changement de son environnement social, économique et familial $»^{53}$.

Ainsi, les critiques féministes du droit dénonçant la non prise en compte des persécutions commises par des particuliers ont effectivement perdu en vigueur car ces violences ne sont plus automatiquement exclues du champ de la définition du réfugié. Il n'en reste pas moins que ces critiques n'apparaissent pas encore totalement dépassées, car l'asile interne tient encore en échec un certain nombre d'entre elles. Un constat tout aussi mitigé peut également être dressé concernant la problématique des motifs de persécution.

\section{B. La problématique des motifs de persécution}

Pour rappel, les textes listent six motifs de persécution susceptibles d'ouvrir droit au statut de réfugié, parmi lesquels

41. Commission des recours des réfugiés (CRR), $1^{\text {er }}$ février 1977, $\mathrm{n}^{\circ} 8637$.

42. CRR, 3 février 1995, nº 273912 .

43. É. Jaillardon, "Quel droit d'asile pour les femmes... ?», in Rapports sociaux de sexe/genre et droit: repenser le droit, L. Langevin (dir.), Paris, Éditions des archives contemporaines, 2008, p. 37-60, spéc. p. 45

44. CE, 27 mai 1983, Dankha, no 42074

45. Voir, par exemple, CRR, 20 mai 1995, n 272728.

46. CRR, 27 mai 1991, n ${ }^{\circ} 173787$.

47. Voir, par exemple, CRR, 26 octobre 1994, $\mathrm{n}^{\circ} 253902$

48. CRR, SR, 12 février 1993, $\mathrm{n}^{\circ} 216617$; CRR, SR, 12 février 1993, $\mathrm{n}^{\circ} 230571$.

49. Ce principe a été dûment rappelé par le Conseil constitutionnel: CC, déc. $\mathrm{n}^{\circ} 2003-485$ DC du 4 décembre 2003 , Loi modifiant la loi $n^{\circ} 52-893$ du 25 juillet 1952 relative au droit d'asile, cons. 5

50. Cour nationale du droit d'asile (CNDA), 29 novembre 2013, $\mathrm{n}^{\circ}$ 13019552. Ces conditions sont issues de la directive 2011/95/UE.

51. CRR, 7 avril $2005, n^{\circ} 501034$.

52. CC, déc. $\mathrm{n}^{\circ} 2003-485$ DC, cons. 21.

53. CNDA, 29 novembre 2013, $\mathrm{n}^{\circ} 13019552$. 
ne figure pas l'identité sexuelle. Ce silence pourrait suggérer que les femmes persécutées ne sont pas éligibles au statut. C'est en tout cas la critique retenue par les théoriciennes féministes du droit, et que les juridictions administratives ont tenté de combattre en interprétant de manière extensive les motifs préexistants (1), et tout particulièrement le motif de l'appartenance à un certain groupe social (2).

\section{La faible mobilisation des motifs «traditionnels "}

Les motifs de la «race», religion et nationalité sont très rarement mobilisés dans le contentieux pour appréhender les persécutions subies par les femmes. Pourtant, il est vrai qu'ils apparaissent à première vue particulièrement pertinents, tant les atteintes aux droits des femmes sont souvent justifiées sur le fondement de considérations ethniques ou religieuses. Est ici en cause le caractère intersectionnel de ces violences. Une «persécution intersectionnelle» est une forme de persécution fondée sur plusieurs motifs mais où seule la combinaison de ces différents motifs est à l'origine de la persécution ${ }^{54}$. Aucun des motifs, pris individuellement, ne permet à lui seul de fonder la persécution. Pour exemple, les femmes maliennes de l'ethnie soninké sont particulièrement exposées aux mutilations génitales. Mais c'est bien ici l'interaction de l'appartenance ethnique et de l'identité sexuelle qui crée le risque. Pris individuellement, ces motifs sont insuffisants pour générer la persécution. Et c'est précisément parce que l'appartenance « raciale», religieuse, ou nationale ne suffit pas, à elle seule, à justifier la persécution que les juges ne la mobilisent pas. En effet, selon eux, il convient de retenir le motif qui a déterminé la persécution ${ }^{55}$. Or précisément ici, elle ne la détermine pas, ou en tout cas pas à elle seule, donc c'est considéré comme insuffisant.

Quant aux motifs politiques (opinions politiques et action en faveur de la liberté), ils sont mobilisés, eux, depuis les années 1990 au profit de militantes des droits des femmes, qu'il s'agisse de droits civils et politiques, ou économiques et sociaux ${ }^{56}$. Mais ces derniers restent toutefois enfermés dans la notion de militantisme, qui limite fortement leur utilité ici. En effet, en l'état de la jurisprudence, la revendication de droits doit nécessairement intervenir en termes généraux et impersonnels et être portée dans l'espace public, que ce soit par une dénonciation dans les médias, la participation à des manifestations ou une structure militante ${ }^{57}$. Par conséquent, une femme qui se borne à dénoncer une atteinte aux droits dans une perspective individuelle, sans visibilité publique, ne pourra pas se voir reconnaître le statut de réfugié sur le fondement d'un motif politique. Cette dernière condition apparaît particulièrement préjudiciable pour les demandeuses, car, justement, elles ne sont pas toujours admises à intervenir dans l'espace public dans leur pays d'origine ${ }^{58}$.

Les motifs «traditionnels» de persécution n'apparaissent ici pas suffisants pour se saisir de l'ensemble des persécutions faites aux femmes. C'est la raison pour laquelle la CRR a eu tout particulièrement recours au motif de l'appartenance à un certain groupe social, un motif très peu mobilisé jusque dans les années 1990.

\section{Le recours au motif de l'appartenance à un certain groupe social}

Dans son arrêt Ourbih, le Conseil d'État a défini un certain groupe social comme un groupe dont les membres partagent une / des caractéristique(s) commune(s), nécessairement fondamentale(s) depuis l'intervention des directives 2004/83/CE et 2011/95/UE, et qui fait l'objet d'une visibilité sociale ${ }^{59}$. Dans ces conditions, on pourrait aisément imaginer un groupe social des femmes. Pourtant, les juridictions administratives s'y refusent. En effet, depuis 1998, la CRR subordonne l'existence de groupes sociaux à leur caractère circonscrit $^{60}$. Les femmes, qui représentent plus de la moitié de la population, ne peuvent donc en former un. C'est en tout cas ce que la CRR a expressément affirmé dans sa décision Hassan du 20 décembre $2004^{61}$. Dans ce contexte, les juridictions administratives françaises ont imaginé des groupes sociaux, plus restreints, pour appréhender les persécutions faites aux femmes: le groupe social des femmes non mutilées ${ }^{62}$, le groupe social des femmes qui refusent de se soumettre à un mariage imposé ou tentent de s'y soustraire ${ }^{63}$, et enfin le groupe social des femmes victimes d'un réseau transnational de traite des êtres humains à des fins d'exploitation sexuelle qui sont parvenues à s'en extraire ou ont entamé des démarches en ce sens ${ }^{64}$. Mais de telles formulations ne permettent

54. Sur l'intersectionnalité, lire: K. Crenshaw, «Demarginalizing the Intersection of Race and Sex: A Black Feminist Critique of Anti-Discrimination Doctrine, Feminist Theories and Antiracist Politics ", University of Chicago Legal Forum, $\mathrm{n}^{\circ}$ 1, 1989, p. 139-167.

55. CNDA, 14 novembre 2013, $\mathrm{n}^{\circ} 12024083$.

56. Par exemple, pour le motif de l'action en faveur de la liberté: CRR, 7 février 2001, $\mathrm{n}^{\circ} 356008$ (pour une Algérienne militant contre les dispositions inégalitaires du Code de la famille); CRR, 24 octobre 2002, no 407759 (pour une Bangladaise en charge d'un planning familial). Pour le motif des opinions politiques, voir aussi: CNDA, 30 avril 2008, nº 599747 (pour une femme militant contre les violences sexuelles en République démocratique du Congo); CRR, 16 juillet 2002, $\mathrm{n}^{\circ} 388131$ (pour une Mauritanienne militant pour l'accès à l'éducation des femmes).

57. A. Korsakoff, Vers une définition genrée du réfugié. Étude de droit français, thèse de doctorat en droit public, université de Caen Normandie, 2018, p. 402-409.

58. T. Spijkerboer, Gender and the Refugee Status, Dartmouth, Ashgate, 2000, p. 58

59. CE, 23 juin 1997, Ourbih, $\mathrm{n}^{\circ} 171858$.

60. CRR, 23 novembre $1998, n^{\circ} 323912$.

61. CRR, 20 décembre 2004, $\mathrm{n}^{\circ} 433535$.

62. CE, Ass., 21 décembre 2012, $\mathrm{n}^{\circ} 332491$.

63. CNDA, 23 juillet 2018, $\mathrm{n}^{\circ} 15031912$.

64. CNDA, 30 mars 2017, $\mathrm{n}^{\circ} 16015058$. 
respectivement de se saisir que des cas de mutilations génitales féminines, de mariages forcés et de prostitution forcée.

Plus encore, pour être mobilisables, ces groupes sociaux doivent faire l'objet d'une visibilité sociale dans le pays d'origine des requérantes. Or, la Cour nationale du droit d'asile retient une définition très restrictive de cette condition, puisqu'elle l'appréhende uniquement par la négative. Il faut en effet que le groupe transgresse une norme sociale, qu'elle soit juridique ou coutumière, et encoure des persécutions présentant un caractère constant, répété et à une échelle suffisamment significative ${ }^{65}$. En d'autres termes, il ne faut pas seulement que la demandeuse au statut soit persécutée, mais également que l'ensemble des femmes placées dans la même situation dans son pays d'origine le soit. Cela introduit donc une distinction parmi les demandeuses suivant leur pays d'origine, qui déterminera si elles peuvent ou non prétendre au statut, sans tenir compte de la réalité de leur situation personnelle.

Ainsi, on est encore bien loin de pouvoir affirmer que la France reconnaît le statut de réfugié aux femmes persécutées. Cela ne concerne que certaines atteintes aux droits: celles découlant d'un militantisme en faveur des droits des femmes, les mutilations génitales, les mariages forcés et les conséquences d'une soustraction à une prostitution forcée. Et encore, dans ces trois derniers cas, seules les femmes de certains pays sont concernées.

La voie de la migration comme remède à une situation de vulnérabilité acquise dans le pays d'origine n'apparaît donc pas toujours assurée, tant la définition du réfugié reste rédigée en des termes restrictifs. Cette remarque vaut pour notre cas d'étude, les femmes persécutées, mais peut être élargie à d'autres problématiques (les homosexuels persécutés, les déplacés environnementaux...). 